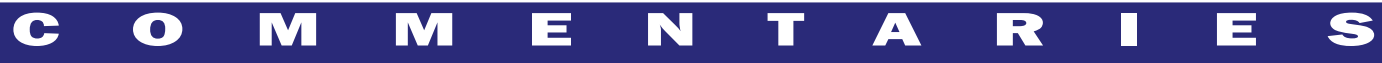

\section{Fertility Massage: an Unethical Practice?}

\author{
Sarah Fogarty, PhD \\ School of Medicine, Western Sydney University, Campbelltown, NSW, Australia
}

\section{INTRODUCTION}

Infertility is defined as not being able to get pregnant after one year of unprotected intercourse (or six months if a woman is 35 or older). ${ }^{(1-3)}$ Women who can get pregnant but are unable to stay pregnant may also be infertile. ${ }^{(1-3)}$ Reported prevalence of infertility rates vary, but most are around $15 \%$ of couples after one year of unprotected intercourse. ${ }^{(2,3)}$ Fertility decreases with age for both men and women, declining around 35 years. ${ }^{(3)}$ For women, by the age of 40 their fertility has decreased significantly and some report this as falling by half. ${ }^{(3)}$ Pregnancy success rates (or conception rates) can be reported by cycle (e.g., the rate per month) or cumulatively (chances of conceiving over a period of time). These rates are very different, and care needs to be taken to ensure clear reporting of rates so that confusion does not occur. Rates are also different for natural conceptions and assisted reproductive conceptions (e.g., in vitro fertilization (IVF)). Reported cumulative natural conception rates are used less in the literature and are based on having vaginal intercourse each month over a 12-month period. Both forms of reporting for natural and assisted conceptions show a similar trend, whereby younger women have a greater chance of conceiving and older women have less chance. ${ }^{(1,2)}$ Infertility can affect the couples' psychological well-being and sexual relationship ${ }^{(4)}$ and cause significant personal anguish. ${ }^{(2)}$ The overwhelming desire to conceive and the psychological stress that accompanies infertility mean that these couples/ individuals are a vulnerable group and, as such, there is an ethical, moral, and legal requirement for professional, open, and truthful promotion, marketing, and advertising about the benefits of infertility treatments. This includes the potential role of massage for the treatment of infertility. Massage specialization seems to be increasing as massage therapists hone their skills in a particular area and as they distinguish themselves from their competitors. One area of specialization is infertility massage. The aim of this commentary is to discuss the evidence, or lack thereof, for infertility massage, the implications for treatment, and the risks and ethical issues associated with the promotion, advertising, and treatment of fertility massage.

\section{MASSAGE AND PSYCHOLOGICAL STRESS- INDUCED INFERTILITY}

There is good evidence showing that stress can affect the reproductive system and thus the ability to conceive. Research has found that psychological stress can affect both men and women, with psychological stress leading to lower serum total testosterone levels with secondary rises in serum luteinizing hormone and follicle-stimulating hormone levels altering seminal quality in men. ${ }^{(5)}$ In women, stress can increase the level of cortisol (a stress hormone) which inhibits estradiol production/biosynthesis by "affecting the granulosa cell functions within the follicle"(6) which leads to a deterioration of the quality of the oocyte and, in IVF, a reduction in the number of retrieved oocytes. ${ }^{(6)}$ Stress can also affect the hypothalamicpituitary-ovarian axis by reducing hypothalamic gonadotropin-releasing hormone secretion, in turn reducing pituitary secretion of luteinizing hormone and follicle-stimulating hormone, thereby reducing ovarian hormone synthesis and, in some cases, ovulation. ${ }^{(7)}$ Psychological stress increases the concentration of glucocorticoids and catecholamines ${ }^{(8)}$ which can excite catecholamines alpha receptors and cause vasoconstriction, thereby reducing uterus blood flow ${ }^{(7)}$ which may reduce endometrial and sub-endometrial blood flow and thus pregnancy outcomes (both in IVF and non-IVF pregnancy attempts). ${ }^{(7)}$ In addition, research has shown that psychological stress can trigger embryonic death. ${ }^{(9-11)}$ The exact mechanism for this loss is unknown, but a decrease in pregnancyprotective cytokines, a reduction in progesterone and prolactin, and elevated glucocorticoids are all thought to contribute to stress-triggered pregnancy loss such as miscarriage. $(9,10,12)$ It is clear that psychological stress can impact the quality of the semen, the quality and quantity of oocytes, ovulation, uterine blood flow, and the mechanisms and processes needed to convince and carry a fetus full-term; however, many individuals endure psychological stress and encounter no problems conceiving or carrying to term. Therefore, the ability to identify individuals who are susceptible to stressinduced reproductive issues is imperative so that these individuals can receive appropriate treatment. 


\section{MASSAGE AND STRESS}

There are a number of studies that show that massage appears to decrease psychological stress, including studies showing moderate pressure massage reduces stress hormones such as cortisol and catecholamine, increases vagal tone, suggestive of a heightened parasympathetic state, and decreases sympathetic nervous activity; (13-17) however, other studies show that massage does not reduce cortisol levels. ${ }^{(18,19)}$ It is noted that light massage does not appear to have the same above-mentioned biochemical effects. ${ }^{(13,14)}$

A 2015 study found that andullation therapy (30min, deep relaxation massage) on an oscillating (vibrating device) prior to blastocyst transfer in a cryocycle (frozen cycle) improved embryo implantation. ${ }^{(20)}$ A frozen cycle (cryocycle) is a cycle that involves the transfer of a thawed, previously frozen embryo to uteri that have been hormonally prepared. The authors hypothesized this was due to a reduction in stress, a reduction in uterine contractions, and an enhancement of blood flow in the abdominal area. ${ }^{(20)}$ The intervention used in the this study is not the traditional 'massage' involving touch; however, this type of massage may activate the body's pressure receptors and thus produce the same type of effect as moderate 'massage' using touch (e.g., hands or feet). Therefore, while the above-mentioned links between the effects of massage for stress and the correlation for its use in stress-related infertility/fertility issues is promising, more evidence is needed.

\section{THE ETHICS OF COMMERCE IN THE PROMOTION OF FERTILITY MASSAGE}

There is an obligation on all massage therapists to ensure that the promotion of any massage service is not unethical. It is important to recognize that ethics encompasses both the act of prescribing or administering an intervention and also the ethics of the selling of health care products and services. The ethical obligations of massage therapists cover both of these aspects as they are providing a service (massage) for sale and administering an intervention. Two of the principles of ethics involved in marketing and selling services of health care are that the product offered "works" (in the language of commercial law, it is "merchantable") and that the product or services are only sold to people who are capable of understanding whether the product will meet their needs. ${ }^{(21)}$ A massage therapist's belief that fertility massage works is not enough to ensure that the promotion of the service being sold is ethical. ${ }^{(21)}$ The foundations of what 'works' in health care is a mixture of clinical experience and judgment, a thorough understanding of human physiology, and a sound understanding of the relevant research evidence. As there is no research available on massage and infertility, and only one paper on improving implantation with vibrational massage, ${ }^{(20)}$ it is difficult to ascertain that fertility massage works. Any promotion of fertility massage that involves an objective effect (i.e., improves fertility, removes scar tissue, or improves egg quality) must be supported by evidence. Any massage therapist offering fertility massage and listing objective benefits of this type of massage will be behaving unethically unless they can provide robust evidence that massage can do these things.

\section{IMPLICATIONS FOR PRACTICE BASED ON THE MASSAGE AND STRESS EVIDENCE}

The moderate pressure massage applied to produce the biochemical and parasympathetic effects for reducing the effects of stress is what would be considered a mainstream, normal massage technique. Therefore, there are no special massage techniques that need to be taught (outside of normal massage therapy training) for psychological stress-related 'fertility' massage.

Evidence is needed for the efficacy of massage for infertility-related stress reduction and more research on how to identify the individuals that psychological stress-reduction massage may be of benefit to. Massage treatment for the purpose of decreasing psychological stress has limitations, with treatment only being able to address the effects of stress not other causes of infertility such as oxidative stress issues, polycystic ovarian syndrome, or primary ovarian insufficiency. Any linkage of benefit to these conditions from massage may be deceptive and misleading.

As there is no specific research on the effect of massage for psychological stress-induced infertility, the dosage (e.g., the number of treatments needed to produce an effect) is not known. The evidence for the reduction in cortisol levels after a massage is mixed, $(13,14,16,18,19)$ and it is not known how long it may take for a drop in cortisol levels to impact and improve reproductive stress-related pathology. How long the effects of massage last are also not known, nor is the most efficacious time in a women's cycle to have massage, nor how long it takes for massage to alter seminal quality in men. Further research is needed to help answer these questions.

How do massage therapists know when stress is related to infertility/fertility issues and which patients may benefit from massage? Fertility issues may involve multiple factors of which stress is just one aspect. To complicate the situation, infertility is stressful and stress might be an outcome from the infertility, not one of the primary causative factors. It is recommended that massage therapists have a referral network in place to help ensure that massage therapy is not used as a primary treatment option for infertile couples. A good working relationship with local 
gynecologists/obstetricians will ensure that a clearer picture of the etiology of the infertility is known and, therefore, the role that massage can play in treatment will also be better defined. There is no research on the role of massage as a prophylactic treatment for reducing the effects of psychological stress-induced infertility. Thus, ethically there is evidence that massage may help with psychological stress and therefore may be of benefit to a stressed individual with fertility issues; however, there is no evidence to use massage prophylactically.

Gynecologists and obstetricians are part of the more traditional health care community where there is a strong ethical and rigorous scientific methodology, which can lead to a gulf between providers of massage and obstetric and gynecological health care providers. Therefore, more rigorous evidence is needed on the effects of massage and stress-related psychological infertility beginning with the presentation of measurable objectives in the form of well-written and strong methodological case studies, case series, and finally a larger controlled trial.

\section{THE DANGERS AND RISKS ASSOCIATED WITH THE PROMOTION AND ADVERTISING OF FERTILITY MASSAGE}

\section{Fertility Massage as a Specialty}

By advertising or promoting or identifying 'fertility' massage as a separate type of massage, the industry is indicating that fertility massage is different to what can be treated in a 'normal' massage consultation. Specialization implies that only a specific type of massage will improve fertility, yet there is no evidence-based research to support this and the 2015 article that found that "massage" improves embryo implantation in a frozen IVF cycle did not use a specialized massage technique. ${ }^{(20)}$ The evidence for how massage may improve fertility is limited to physiological stress $(13,14,17,20)$ and does not include specialized massage techniques to induce benefits. Advertising or promoting fertility massage as a separate type of massage implies to both consumers and other health care professionals that there are no fertility benefits from an 'ordinary/regular' massage treatment and/or that there are more benefits from having a 'fertility massage' than a 'regular massage'.

\section{Ensuring that Statements About the Effects and Benefits of Fertility Massage Are Not Misleading or Deceptive}

Statements made about fertility massage to treat particular conditions and its benefits need to be backed up by acceptable evidence. When promoting or advertising massage to improve fertility, it needs to be really clear what the evidence is saying. This includes for what condition it may provide benefit, for whom it may benefit, and where it will not provide benefit. For example, to say that fertility massage promotes the production and quality of the egg and sperm is misleading. There is some evidence that suggests that deep relaxation vibrational massage may improve embryo implantation ${ }^{(20)}$ and some evidence that massage may aid in decreasing cortisol levels ${ }^{(14,20)}$ in women who are suffering from psychological stress who have increased cortisol levels that lead to a deterioration of the quality of the oocyte and, in IVF, a reduction in the number of retrieved oocytes. ${ }^{(6)}$ There is, however, no evidence that massage can improve egg quality or quantity in women with low ovarian reserves or premature ovarian failure or in women with damaged eggs from smoking, radiation therapy or chemotherapy.

\section{Unreasonable Expectations of Beneficial Treatment}

As there is no direct evidence of efficacy of massage for infertility, any advertisement or promotion of fertility massage needs to ensure that no statements are used that give an unreasonable expectation of the treatment. Therefore, no advertisement or promotion of fertility massage should imply that it was the massage that led to the conception and/or a live birth. Furthermore, it would also be unethical to cite pregnancy success rates associated with massage. There is no evidence linking massage to increased conception and, as pregnancy success rates vary by age, decreasing as the couple ages, ${ }^{(3)}$ it is very easy for an advertisement citing pregnancy success rates to confer unreasonable expectations of benefit. Any promotion or advertising of massage's success in helping couples conceive is indicating unreasonable expectations of benefit, as there is no evidence that massage is a significant contributory factor in conceiving and it is much more likely that the conception success was due to time as cumulative conception rates show, the more than a couple tries to conceive, the more likely they are to succeed. ${ }^{(2)}$

\section{CONCLUSION}

Couples suffering from infertility are a vulnerable group and, as such, must be protected from unethical, misleading, and deceptive advertising and promotion of the benefits of fertility massage. To date, there is no evidence that fertility massage is beneficial and, therefore, any promotion of objective fertility outcomes that would be gained from receiving a fertility massage would be deemed unethical. Any treatment and advertising or promotion of massage must be evidence-based, and practitioners must ensure that the information provided does not mislead, deceive or overstate the benefits of massages. In particular, 
the linking of massage to pregnancy success should be avoided. Massage industry stakeholders, such as massage associations, massage therapists, and researchers, need to develop guidelines and codes of practice around fertility massage, and provide support and education on the ethical and professional promotion of fertility massage.

\section{COPYRIGHT}

Published under the CreativeCommons AttributionNonCommercial-NoDerivs 3.0 License.

\section{REFERENCES}

1. Jansen R. Getting pregnant. A compassionate resource to overcoming infertility and avoiding miscarriage. Crows Nest, Australia: Allen and Unwin; 1997.

2. Chambers GM, Paul RC, Harris K, Oisin Fitzgerald, Clare V Boothroyd, Luk Rombauts, et al. Assisted reproductive technology in Australia and New Zealand: cumulative live birth rates as measures of success. Med J Aust. 2017;207(3):114-18.

3. Dunson DB, Colombo B, Baird DD. Changes with age in the level and duration of fertility in the menstrual cycle. Hum Reproduc. 2002;17(5):1399-403.

4. Luk BHK, Loke AY. The impact of infertility on the psychological well-being, marital relationships, sexual relationships, and quality of life of couples: a systematic review. J Sex \& Marital Ther. 2015;41(6):610-25.

5. Bhongade MB, Prasad S, Jiloha RC, Ray PC, Mohapatra S, Koner BC. Effect of psychological stress on fertility hormones and seminal quality in male partners of infertile couples. Andrologia. 2015;47(3):336-42.

6. Prasad S, Tiwari M, Pandy A, Shrivastav TG, Chaube SK. Impact of stress on oocyte quality and reproductive outcome. J Biomed Sci. 2016;23:36.

7. Dong Y, Cai Y, Zhang Y, Xing Y, Sun Y. The effect of fertility stress on endometrial and subendometrial blood flow among infertile women. Reproduc Biol Endocrinol. 2017;15:15.

8. Engert V, Vogel S, Efanov S, Duchese A, Corbo V, Ali N, et al. Investigation into the cross-correlation of salivary cortisol and alphaamylase responses to psychological stress. Psychoneuroendocrino. 2011;36(9):1294-302.
9. Arck PC, Rose M, Hertwing K, Hagen E, Hildebrandt M, Klapp BF. Stress and immune mediators in miscarriage. Hum Reproduc. 2001;16(7):1505-11.

10. Joachim RA, Hildebrandt M, Oder J, Klapp BF, Arck PC. Murine stress-triggered abortion is mediated by increase of $\mathrm{CD}^{+}$TNF-xxa ${ }^{+}$decidual cells via Substance P. Am J Reproduc Immunol. 2001;45(5):303-09.

11. Parker VJ, Douglas AJ. Stress in early pregnancy: maternal neuro-endocrine-immune responses and effects. $J$ Reproduc Immunol. 2010;85(1):86-92.

12. Xu Z, Zhao J, Zhang H, Ke T, Xu P, Cai W, et al. Spontaneous miscarriages are explained by the stress/glucocorticoid/lipoxin A4 axis. J Immunol. 2013;190(12):6051-58.

13. Field T. Touch for socioemotional and physical well-being: a review. Development Rev. 2010;30(4):367-83.

14. Field T, Hernandez-Reif M, Diego M, Schanberg S, Kuhn C. Cortisol decreases and serotonin and dopamine increase following massage therapy. Int J Neurosci. 2005;115(10):1397-413.

15. Noto Y, Kudo M, Hirota K. Back massage therapy promotes psychological relaxation and an increase in salivary chromogranin A release. J Anesthesia. 2010;24(6):955-58.

16. Lindgren L, Rundgren S, Winso O, Lehtipalo S, Wiklund U, Karlsson M, et al. Physiological responses to touch massage in healthy volunteers. Autonomic Neurosci. 2010;158 (1-2):105-10.

17. Field T. Massage therapy effects. Am Psychol. 1998;53(12): 1270-81.

18. Moraska A, Pollini RA, Boulanger K, Brooks MZ, Teitlebaum L. Physiological adjustments to stress measures following massage therapy: a review of the literature. Evidence-based Complement Alt Med. 2010;7(4):409-18.

19. Moyer CA, Seefeldt L, Mann ES, Jackley LM. Does massage therapy reduce cortisol? A comprehensive quantitative review. J Bodywork Movement Ther. 2011;15(1):3-14.

20. Okhowat J, Murtinger M, Schuff M, Wogatzky J, Spitzer D, Vanderzwalmen $\mathrm{P}$, et al. Massage therapy improves in vitro fertilization outcome in patients undergoing blastocyst transfer in a cryo-cycle. Altern Ther Health Med. 2015;21(2):16-22.

21. MacDonald C, Gvura S. Alternative medicine and the ethics of commerce. Bioethics. 2016;30(2):77-84.

Corresponding author: Sarah Fogarty, $\mathrm{PhD}, \mathrm{PO}$ Box 8218, Ferntree Gully, Victoria, Australia 3156

E-mail: doctorfogarty@gmail.com 